\title{
Editorial
}

\section{Innovation resuscitation and management in FM and operations management}

\author{
Journal of Retail and Leisure Property (2008) 7, 163-166. \\ doi:10.1057/rlp.2008.17
}

In the late 1990s, I wrote much about the changing nature of operations management and how it would and should change to accommodate the changing needs of business. I focused primarily on airport management and the nature of the low-cost airlines. Client organisations' demands are, of course, always changing as business changes and as the availability of services changes. As more operations and services management organisations enter the market, often basing their product upon limited previous experience, so the challenge for the next few years will be adding value to business through product differentiation enabled by bespoke, integrated and innovative delivery.

One of the problems that I experienced in carrying out research over the last ten years is that there appears to be something of a misconception about what constitutes innovation and how innovation management actually works. Figure 1, developed by Goyal at Liverpool John Moores University, models the generic innovation cycle within most organisations. This provides a reference point for the active management of innovation. It serves as a reminder that innovation has basic drivers but that its effective management for competition through product differentiation relies upon the identification and stimulation of the micro drivers (which are not shown). At this level this model is fairly easy to understand but the new 'Resuscitation Model' which for the first time reveals the underlying organisational innovation drivers has allowed US to develop a method for delivering precision change into an organisation to deliver a fast tracked facilities management (FM) and operations management improvement. We work closely with many organisations across the world. One of the companies involved in the development of the new product is MITIE Managed Services. Andrew Bovill, Business Development Manager at MITIE Managed Services told me recently that '....we recognise that stimulating and managing innovation in service delivery is essential to maximising added value for the client organisation. In order to do this effectively we address issues of both process and culture in any services planning operation'. In other words, in order to manage innovation you must have innovation to manage in the first place and this will certainly mean addressing cultural issues in addition to process re-engineering. The innovation resuscitation product delivers a bespoke prescribed set of changes to an organisation and provides a 


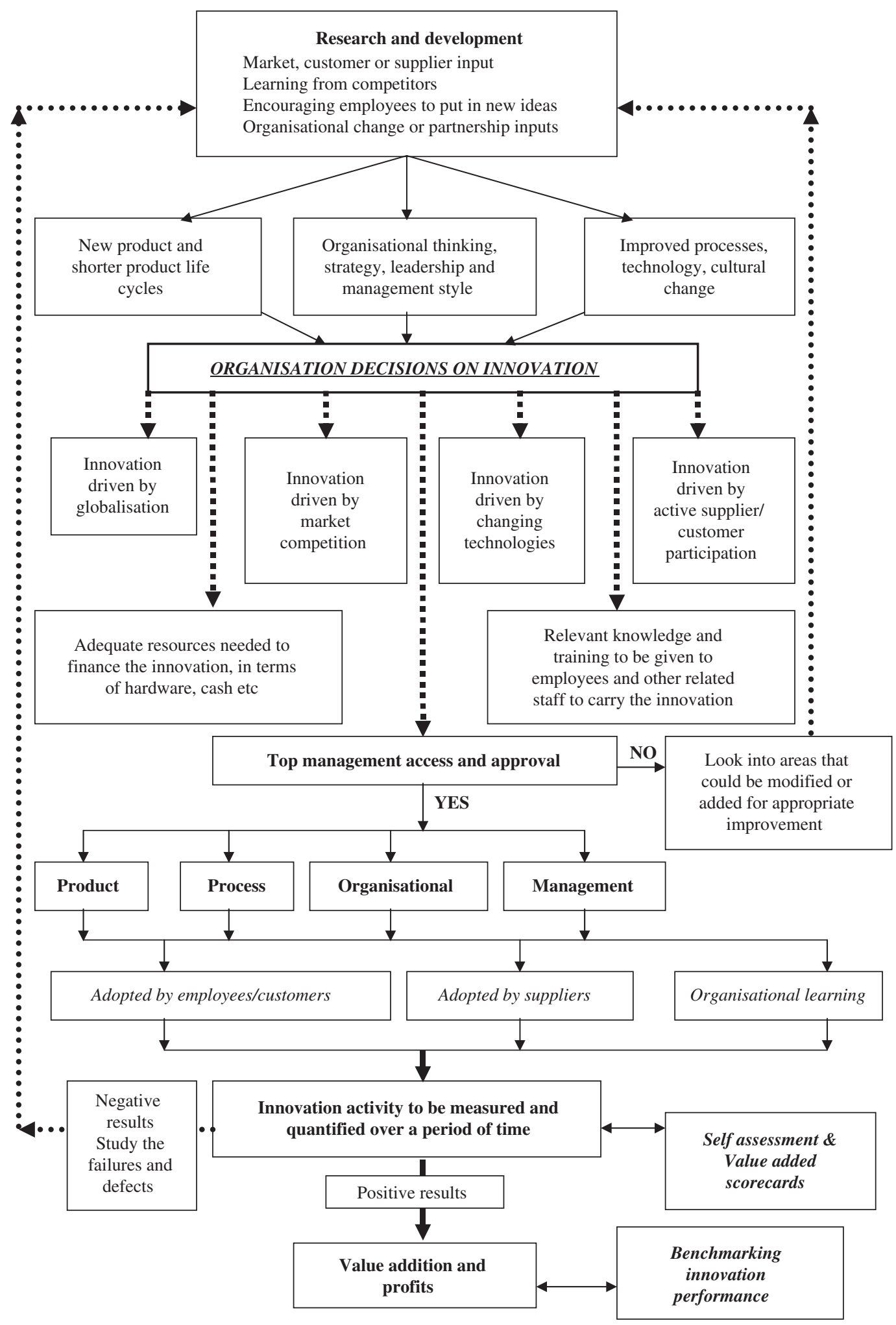

Figure I: Generic Innovation Model

Source: Goyal, 2008 
management structure to accommodate the changes. The new model identifies process nodes that become the focus for resource input and change within the organisation. It changes the company DNA as well as its external and internal interaction.

There are, however, certain prerequisites for successful stimulation and management of innovation.

\section{OUTLOOK AND VISION}

For innovation to be successful it requires change. There will be associated risk and often a degree of uncertainty within an organisation. The drivers of innovation are a key motivator in compelling the organisation to develop the processes, systems, to allocate the resources and the culture needed to support innovation.

\section{CUSTOMER FOCUSED ADDED VALUE}

The purpose of innovation is to find better ways to surprise and delight customers and to create additional value within the organisation. Value is defined and measured in terms of core outputs and must not be solely benchmarked against similar services.

\section{OPENNESS}

It is only in an environment of mutual trust and respect that an organisation can develop a truly innovative approach to any aspect of its operations. There must be a cultural fit between the organisations and within the project team. This will provide for good and honest communication without hidden agendas and organisational loyalty.

\section{CREATIVE THINKING}

Innovation requires creative thinking and an ability to see routes outside the normal scope of a project. New possibilities should constantly be sought and the skills of the organisational innovators should be highly valued. Capturing an innovation project in the form of a plan is therefore often problematic. The hard working company man will almost certainly be the wrong person to manage innovation as the rigidity that serves well daily may sound the death knell for innovation.

\section{A diverse, information and interaction-rich environment}

The ideal scenario is to have people with different perspectives, working together toward a common objective, with accurate, up-to-date information and the proper tools.

\section{Tolerance of risk}

The creation of anything new involves risk and the possibility of failure.

- An innovative environment honours efforts that did not work (AKA failures) as learning experiences and part of the innovation process. 


\section{Reward the individual}

Everyone should own innovation and should be included in its development. The Royal Bank of Scotland Inncuvator scheme is illustrative of a dynamic organisation seeking to involve employees at all levels.

Finally there will always be resistance to new ways of working. It is necessary to manage this element of change as with any other business change.

Michael Pitt Managing Editor 